\title{
Applications of Semiconductor Lasers to Secure Communications
}

\author{
Claudio R. Mirasso \\ Departament de Física, Universitat de les Illes Balears, E-07071 Palma de Mallorca, Spain
}

\begin{abstract}
We numerically study the synchronization of two chaotic semiconductor lasers in a master-slave configuration. To synchronize the lasers a small amount of the output power from the master laser is injected into the slave laser. We show that the output of the master laser can be used as a chaotic carrier to encode a digital message which can be recovered at the receiver. We also check the quality of the synchronization diagram when the two lasers are slightly different.
\end{abstract}

\section{INTRODUCTION}

The world is currently witnessing an ever-growing demand for communications services. This is being supported by a rapid increase in transmission and switching capacity in networks of all kinds. Two major issues in these networks are privacy and security. Although software codification has proven to be useful for data encoding, the continuous increase of computer speed threatens the safety of this procedure. A contribution to solve this problem is by additionally encoding at the physical level using chaotic carriers generated by components operating in the non-linear regime.

Semiconductor lasers are ideal candidates for the realization of these non-linear transmitter and receiver systems because they are already inherently non-linear devices that under various operation conditions exhibit non-linear dynamical behaviour associated with, e.g., fast irregular pulsations of the optical power or wavelength hopping. In the today existing and operating communication schemes these types of behaviour are not desired and sometimes tremendous efforts have to be performed to avoid them. It is our particular aim to explicitly benefit from these behaviours, usually called optical chaos.

The essence of the optical chaotic communication resides in the fact that two spatially separated chaotic lasers, in our case semiconductor diode lasers, can synchronize to each other. Synchronization means that the irregular time evolution of the emitter laser, either in the optical power $\mathrm{P}$ or in the wavelength, can be perfectly reproduced by the receiver laser; see Fig. 1 . 

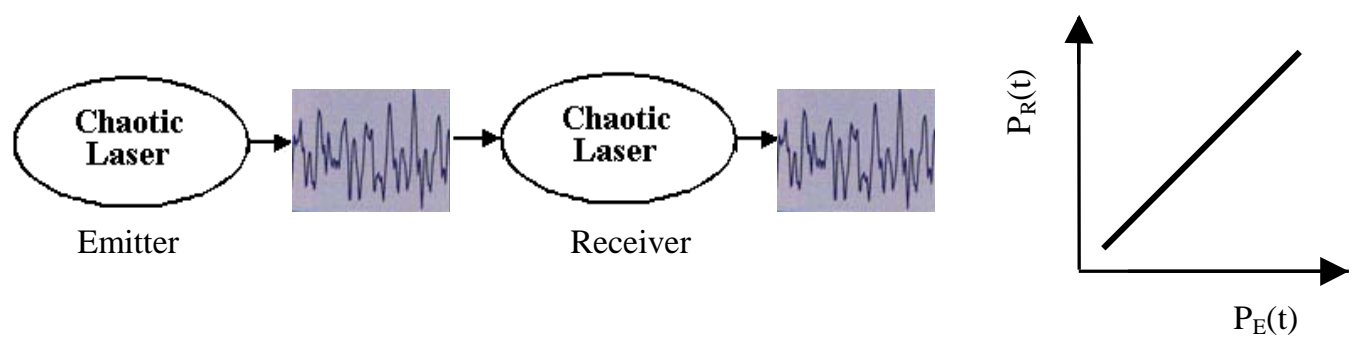

FIGURE 1. Schematic representation of synchronization. The synchronization diagram, i.e. the evolution of output signal of the emitter vs. the output signal of the receiver, is plotted at the right side. The $45^{\circ}$ line represents perfect synchronization.

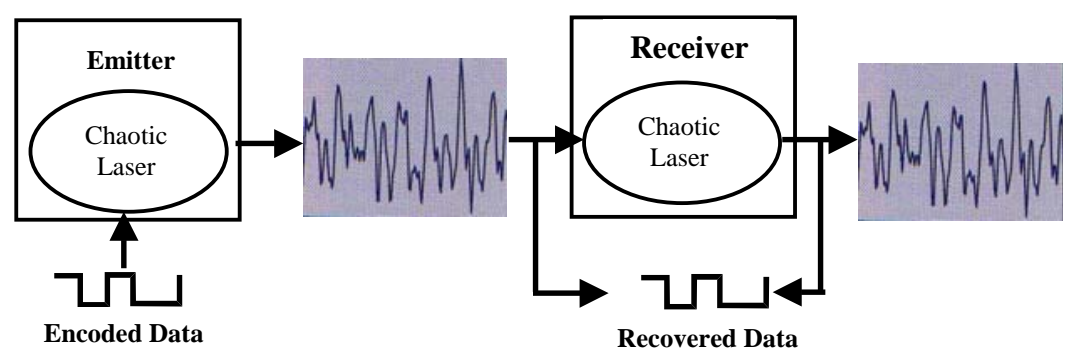

FIGURE 2. Schematic representation of the encoding/decoding process

Once the two lasers have been synchronized by a suitable method, we can use the chaotic output of the transmitter laser as the carrier on which the message is encoded. The other laser, at the receiver, allows the message to be extracted. Decoding is based on the non-linear phenomenon of chaos synchronization between emitter and receiver [1]. The key issue resides in the fact that the receiver synchronizes to the chaotic oscillations of the emitter (the carrier) suppressing the encoded message. Therefore, by comparing the input (carrier + message) and output (carrier only) of the receiver the message can be extracted (see Fig. 2).

The key for effective synchronization (and thus for decoding) lies in the use of very similar components for both chaotic systems, with close matching with respect to parameters and operation conditions. Decoding without the right receiver is very difficult to achieve due to the high frequencies involved and the large number of dynamical degrees of freedom of the chaotic carrier. It is worth noting that chaotic carriers provide in semiconductor lasers a broad spectrum (typically in the $\mathrm{GHz}$ range) where the message is hidden. The properties of these carrier signals, and the way the message is encoded, are such that with a linear filtering process it is not possible to extract the message. Also correlators and frequency-domain analysis would fail. 


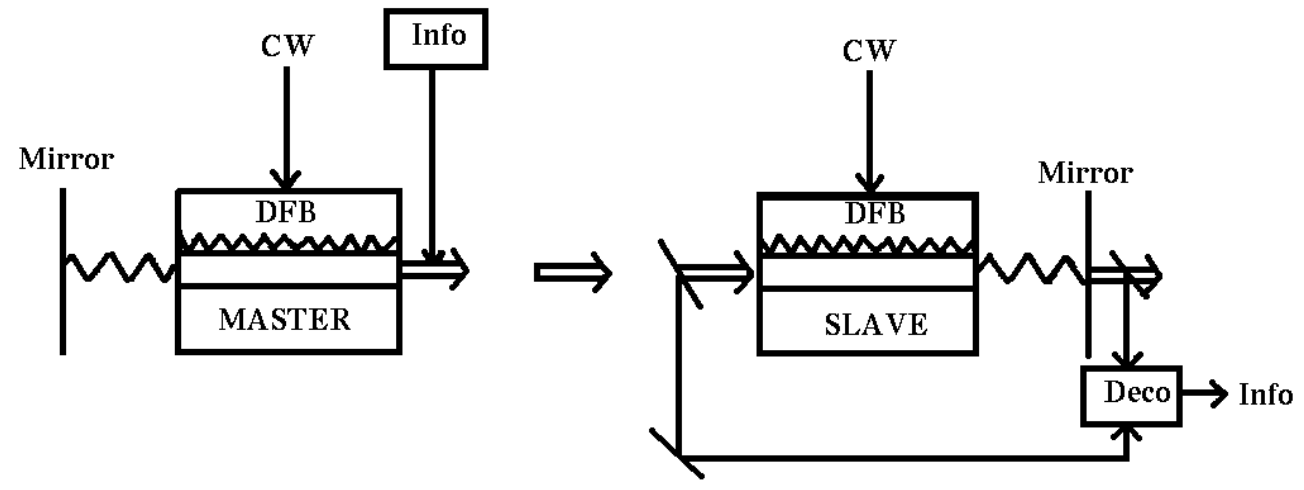

FIGURE 3. Proposed set-up for chaos synchronization and message encoding using semiconductor lasers (similar to the one proposed in Refs. [5,6])

The idea of communicating using transmitters and receivers operating in the nonlinear dynamical regime has recently attracted the attention of many researchers. Different chaotic synchronization schemes and their applications to encoded communications have been proposed. This was first studied using electronic circuits [2] and it has been more recently proposed in solid state [3], fibre ring lasers [4], semiconductor lasers [5,6,8-15] and microchip lasers [16].

Chaotic signals have similar features as those used in broad spectrum communications and related fields where information is hidden within complex codes or noisy signals. Up to the present, the methods for generating chaotic signals have generally used simple electrical circuits. However, there have been two main problems with this approach. Firstly, most of the circuits have been designed in the audio range with message bandwidths limited to about $10 \mathrm{kHz}$. Although $\mathrm{RF}$ circuits may be contemplated, it is difficult to attain the multi-GHz frequencies required in many communication channels. Furthermore, most already installed high speed communication networks are based on optical fibers. Therefore, a codification scheme directly based on an optical carrier is highly desirable. The second more fundamental problem of the chaotic carrier generated electronically is its lowdimensionality, which results in a low level of confidentiality when applied to secure communication transmissions. Both these issues are overcome by the proposed use of high-dimensional chaos generated by lasers subject to feedback, either all optical or electro-optical.

Recent studies have shown the feasibility of synchronizing the so-called hyperchaos, i.e. attractors with a very large number of degrees of freedom, in optical systems. A set of two semiconductor lasers subjected to optical feedback, operating in the chaotic regime, and linked through an optical fiber were numerically studied in Refs. [5,6]. The two lasers were coupled as in Fig. 3, the scheme that will be followed during the rest of the paper. The master laser (ML) operates in the coherence collapse regime due to the optical feedback, i.e. the output power oscillates 
chaotically; see also the chapter by Fischer et al. The message to be encrypted can be added at the output of the ML (chaos masking) or included in the injection current (chaos shift keying). The slave laser (SL) is similar to the ML and is subjected to the same feedback. When the ML and SL are not connected, their output power are uncorrelated and the synchronization diagram (a plot of the ML output vs. the SL output) exhibits a cloud of points, as will be shown later. However, when a small amount of light coming from the ML is injected into the SL the two subsystems are able to synchronize and an almost $45^{\circ}$ line in the synchronization diagram is obtained. As it was already mentioned, the SL laser synchronizes to the carrier of the ML by filtering the message. Under such conditions the message can be recovered by processing the input and output of the SL.

Another common way to produce a chaotic output from a semiconductor laser is by optical injection. Optical injection allows for different kind of routs to chaos and types of instabilities. A carefully study of this system can be found in Ref. [7]. Synchronization of semiconductor lasers with an injected signal operating in the chaotic regime was very recently studied by Chen and Liu [8], although the original idea was proposed by V. Annovazzi and coworkers [9], to encrypt information. They proposed a set-up that is shown in Fig. 4 of Ref. [9]. In the system, the transmitter is switched between two different chaotic orbits by modulating the injection current of the laser by the bit stream to be transmitted. Decoding at the receiver is performed with two replicas of the emitter: the first is biased with a current $\left(J_{0}\right)$ corresponding to the bit " 0 " and the second at a bias $\left(J_{1}\right)$ corresponding to the bit " 1 ". The received signal is sent to both decoders, however only the one having the pump current corresponding to the transmitted bit synchronizes, as shown in Fig. 5 of Ref. [9].

Encryption of information within a chaotic carrier was experimentally demonstrated by van Winggeren and Roy [4] using two similar chaotic erbium doped fibre ring lasers (EDFRL). The set-up is shown in Fig. 1 of Ref. [4]. A coupler injects the message to be encrypted into the first EDFRL. The output is extracted by another coupler and directed to the second EDFRL (the receiver). Both EDFRL at the emitter and receiver were fabricated to match as closely as possible. With an appropriate decoding process they were able to encode/decode a digital message at a bit rate up to $250 \mathrm{Mbit} / \mathrm{s}$. Moreover, they showed the possibility of transmitting the message up to $35 \mathrm{~km}$ of single mode dispersion shifted optical fibers [4].

Almost at the same time, a different approach to encode information was proposed and experimentally demonstrated by J. P. Goedgebuer and coworkers [11]. They proposed a method for encrypting a signal within the high dimensional chaotic fluctuations of the wavelength from a delayed feedback tunable laser. The novelty of the idea was not only to use for the first time chaos in wavelength, but also a non-linear electro-optical feedback was included to generate the chaos (see Fig. 1 of Ref. [11]). This non-linear electro-optical feedback guarantees a large dimension of the generated chaos (between 100-1000), also called hyperchaos, which is very important for the security of the encrypted message. They also performed message encoding/decoding of a sine wave, although at a quite low rate $(\mathrm{KHz})$. 
One year later, Sivaprakasam and Shore demonstrated experimentally the synchronization of two external-cavity semiconductor laser diodes, in the configutration shown in Fig. 3. They found an optimal coupling strength between the ML and the SL for which a good synchronization is obtained (see Fig. 2 and 3 of Ref. [12]). In a later paper they showed the possibility of encoding/decoding a square wave signal, however at a low bit rate (KHz).

The first demonstration of GHZ signal encoding/decoding within a chaotic carrier was performed by I. Fischer and coworkers [13]. They used a similar scheme as the one shown in Fig. 3 but for the slave laser they removed the optical feedback, i.e. the SL operates under continuous wave. Although one could think that this system would not be useful for encoding and decoding information, they showed that the receiver synchronizes to the chaotic output of the transmitter (as shown in Figs. 2 and 3 of Ref. [13]) suppressing the encoded message, as it is clearly shown in Fig. 4 of Ref. [13]. By operating with the input and output of the receiver they were able to reconstruct the encoded signal.

In this work we consider a model for chaos synchronization and digital communications using as a carrier the chaotic output of a single-mode semiconductor laser subject to external optical feedback (Master Laser, ML), as shown in Fig. 3. We encode a digital message within the chaotic output of the master laser by either a time dependent attenuation of the carrier (chaos masking), in a scheme similar to the one proposed in Refs. [5,6], or by slightly modulating the injection current (as in Ref. [9]) (chaos shift keying). After codification, the carrier containing the message is injected into the receiver. For simplicity, we will consider a free space link, although transmission through dispersion shifted optical fibers has been shown to be feasible [6]. The receiver, the Slave Laser (SL), is also a single-mode semiconductor laser with external feedback, with parameters similar to the ML and operating under similar conditions. The two lasers display chaotic behavior with different time traces if they operate separately, i.e. with no-coupling between them. Even for identical initial conditions the presence of spontaneous emission noise will make time traces to depart from each other in time. However, if a small amount of the ML power is injected in the SL they can synchronize. This synchronization will allow us to encrypt messages within the chaotic output of the emitter.

The paper is organized as follows. We first describe the model we use for the transmitter and the receiver. Next we present numerical results for both synchronization and message encoding/decoding. Finally, we give a summary and the conclusions of our work.

\section{THE MODEL}

We model the transmitter (ML) and the receiver (SL) by using the rate equations for the complex slowly varying amplitude of the electrical field $E_{t, r}$ and carrier number inside the cavity $N_{t, r}[5]$ (t,r stand for transmitter and receiver respectively). We also include spontaneous emission noise in the field equation. The equations 
read:

$$
\begin{gathered}
\frac{d E_{t, r}(t)}{d t}=\left(1+j \alpha_{t, r}\right)\left(G_{t, r}(t)-\frac{1}{\tau_{t, r}}\right) \frac{E_{t, r}(t)}{2}+\gamma E_{t, r}(t-\tau) e^{j \omega_{t, r} \tau} \\
+\kappa_{r} E_{e x t}(t) e^{-j \Delta \omega t}+\sqrt{2 \beta N_{t, r}(t)} \xi_{t, r}(t) \\
\frac{d N_{t, r}(t)}{d t}=\frac{I}{e}-\frac{1}{\tau_{n_{t, r}}} N_{t, r}(t)-G_{t, r}(t)\left|E_{t, r}(t)\right|^{2} \\
G_{t, r}(t)=\frac{g\left(N_{t, r}-N_{o t, r}\right)}{\left(1+s\left|E_{t, r}(t)\right|^{2}\right)}
\end{gathered}
$$

We consider the transmitter $(t)$ and the receiver $(r)$ to be very similar lasers, so we take, in principle, the same parameters for them: $\mathrm{g}=1.5 \times 10^{-8} \mathrm{ps}^{-1}$ is the gain parameter, $\mathrm{s}=5 \times 10^{-7}$ is the gain saturation coefficient, $\alpha=5$ is the linewidth enhancement factor, $\beta=1.1 \times 10^{-9} \mathrm{ps}^{-1}$ is the spontaneous emission rate, $e=$ $1.602 \times 10^{-19} \mathrm{C}$ is the electronic charge, $\tau_{n}=2 \mathrm{~ns}$ is the carrier lifetime, $\tau_{t, r}=2 \mathrm{ps}$ is the photon lifetime, $N_{o}=1.5 \times 10^{8}$ is the carrier number at transparency, $\gamma=30$ $\mathrm{ns}^{-1}$ is the feedback coefficient, $\tau$ is the external cavity roundtrip time and $\Delta \omega$ is the detuning between the optical frequencies of the free-running ML and SL. $I=44$ $\mathrm{mA}$ is the bias current (the threshold current is $I_{t h} \approx 14.7 \mathrm{~mA}$ ) and $\omega_{t, r} \approx 1.2 \times 10^{3}$ $\mathrm{ps}^{-1}$ is the angular frequency of both ML and SL under continuous wave operation,. The random spontaneous emission process is modeled by a complex Gaussian white noise term $\xi(t)$ of zero mean and correlation $<\xi_{a}(t) \xi_{b}^{*}\left(t^{\prime}\right)>=2 \delta_{a b} \delta\left(t-t^{\prime}\right)$ where $\mathrm{a}, \mathrm{b}$ stand for $t, r$. The term $\kappa_{r} E_{\text {ext }}$ in (1), which takes into account the signal generated by the ML, exists only for the receiver laser. The field $E_{\text {ext }}$ is the input signal at the receiver and $\kappa_{r}$ is the coupling parameter of the injected field into the SL. Equation (1)-(3) are written in the reference frame where the emission frequency is zero when neglecting spontaneous emission and the laser is biased at threshold.

\section{NUMERICAL RESULTS}

\section{Synchronization}

A typical time trace and optical spectrum for the ML and SL operating in the chaotic regime are displayed in Fig. 4; see also the chapter by Fischer et al. In the time trace it can be clearly seen large intensity fluctuations indicating a well established chaotic regime, and consequently a broad optical spectrum of some tens of $\mathrm{GHz}$ width. The parameters used in these simulations, and the ones that we will use for the rest of the paper, are those given in the previous section. In Fig. 4 $\tau=0.3 \mathrm{~ns}$ and $\kappa_{r}=50 \mathrm{~ns}^{-1}$. 

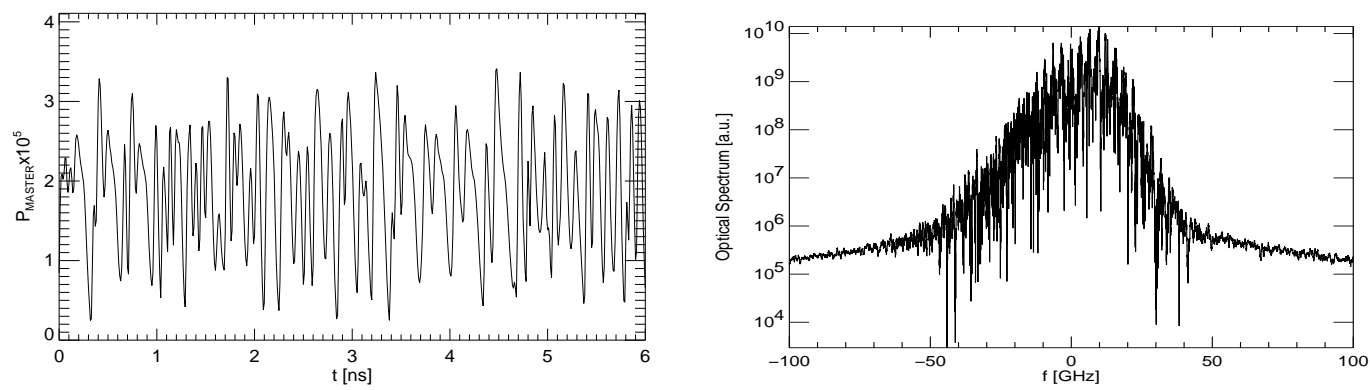

FIGURE 4. Typical time trace for a semiconductor laser subjected to optical feedback operating in the coherence colapse regime (left), and its optical spectrum (right). $\kappa_{r}=50 \mathrm{~ns}^{-1}, \Delta \omega=0$ $\tau=0.3 \mathrm{~ns}$ and the rest of the parameters are those given in the previous section.

For identical laser parameters and operating conditions the synchronization diagram, i.e. the output power of the SL vs. the output power of the ML, would exhibit an almost $45^{\circ}$ straight line. However, a small deviation is obtained due to the fact that the SL has an extra power coming from the light injected from ML. By taking slightly different photons lifetime this difference can be compensated. The quality of the synchronization between the ML and SL laser strongly depends on some parameters as coupling coefficient, noise, feedback strength, etc. The robustness of the synchronization scheme was partially studied in Ref. [6] were the internal parameters of the ML and SL were varied. Through this section we will analyse the effects of some other parameters involved in the scheme in some detail.

We first examine the dependence of the synchronization quality with respect to the coupling coefficient. A simple expression for this coefficient, valid for FabryPerot semiconductor lasers, can be given: $\kappa_{r}=\sqrt{1-R} \eta_{\text {ext }} /\left(\tau_{L} \sqrt{R}\right)$ where $R$ is the facet power reflectivity of the SL, $\tau_{L}$ is the cavity roundtrip time of the light within the laser and $\eta_{\text {ext }}$ accounts for losses different than those introduced by the laser facet. For our calculations we take $\tau_{L}=6.6 \mathrm{ps}$ (which corresponds to a laser length of $\sim 250 \mu \mathrm{m}$ ) and $R=30 \%$. The different values of $\kappa_{r}$ are obtained by considering different values of $\eta_{\text {ext }}<1$. It is evident that a minimum value for the coupling between the ML and SL is required in order to reach good synchronization. Even for the case of identical lasers and operating conditions a small coupling would not allow for synchronization. The synchronization diagram as a function of the coupling strength $\left(\kappa_{r}\right)$ is shown in Fig. 5. When the two lasers are decoupled, their trajectories depart each other as time evolves and the synchronization map becomes a cloud of points with no correlation between ML and SL outputs (Fig. 5 a). However, this situation may change if a small amount of the light emitted by the ML is injected into the SL. Even for small amounts of coupling the synchronization diagram presents clear signs of synchronization between ML and SL. It can be clearly seen in the figure that the synchronization improves for increasing $\kappa_{r}$. For our parameters values, $\kappa_{r} \gtrsim 50 \mathrm{~ns}^{-1}$ starts to give a good synchronization diagram. 

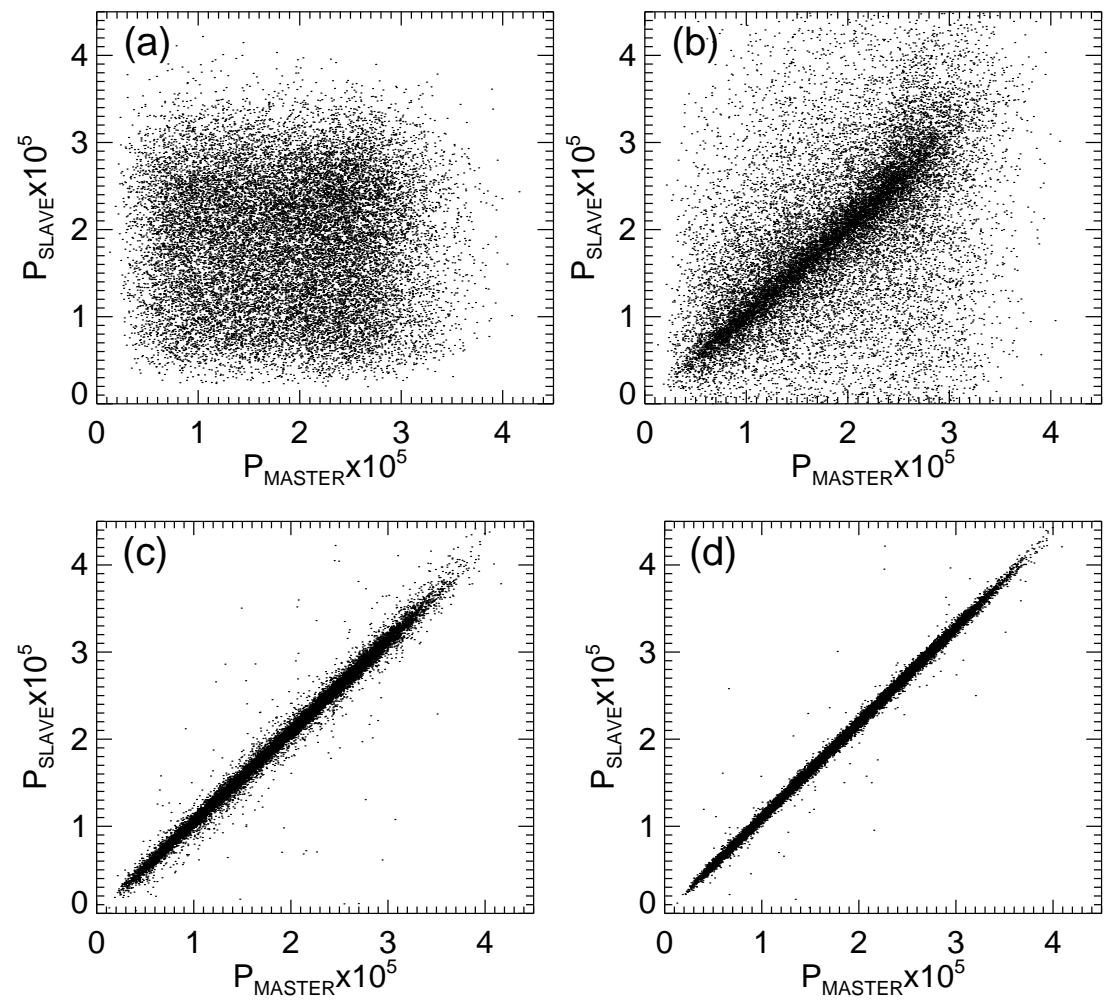

FIGURE 5. Synchronization diagram for different values of $\kappa_{r}$, namely $\kappa_{r}=0$ (a), $\kappa_{r}=25$ $\mathrm{ns}^{-1}(\mathrm{~b}), \kappa_{r}=50 \mathrm{~ns}^{-1}$ (c), and $\kappa_{r}=100 \mathrm{~ns}^{-1}$ (d). $\Delta \omega=0, \tau=0.3 \mathrm{~ns}$ and the rest of parameters are those given in the previous section

In practice, it is not possible to find pairs of identical semiconductor lasers. Variations of internal parameters have been partially studied in [6], as was already mentioned. In the this article, the photon lifetime was identified as the most critical parameter and changes in its value larger than $\sim 5 \%$ could yield to a loss of synchronization. Synchronization quality would be also strongly influenced by, e.g., noise effects. Several noise sources would be present in real communication systems, including spontaneous emission noise in diode lasers, current noise, amplified spontaneous emission noise, in case of optical amplification, etc. In Fig. 6 we show how the synchronization degrades with the spontaneous emission factor $\beta$ of the lasers. It is evident that for low values of $\beta$ a good synchronization diagram is observed but it strongly degrades for larger values of the noise level, until the signals become almost uncorrelated. These results indicate that noise sources should be very well controlled in real systems.

Another sensible parameter is the detuning. It accounts for the difference between the free-running laser frequencies of the ML and SL. Fig. 7 shows the synchronization diagram as a function of the detuning. This effect was studied in detail 

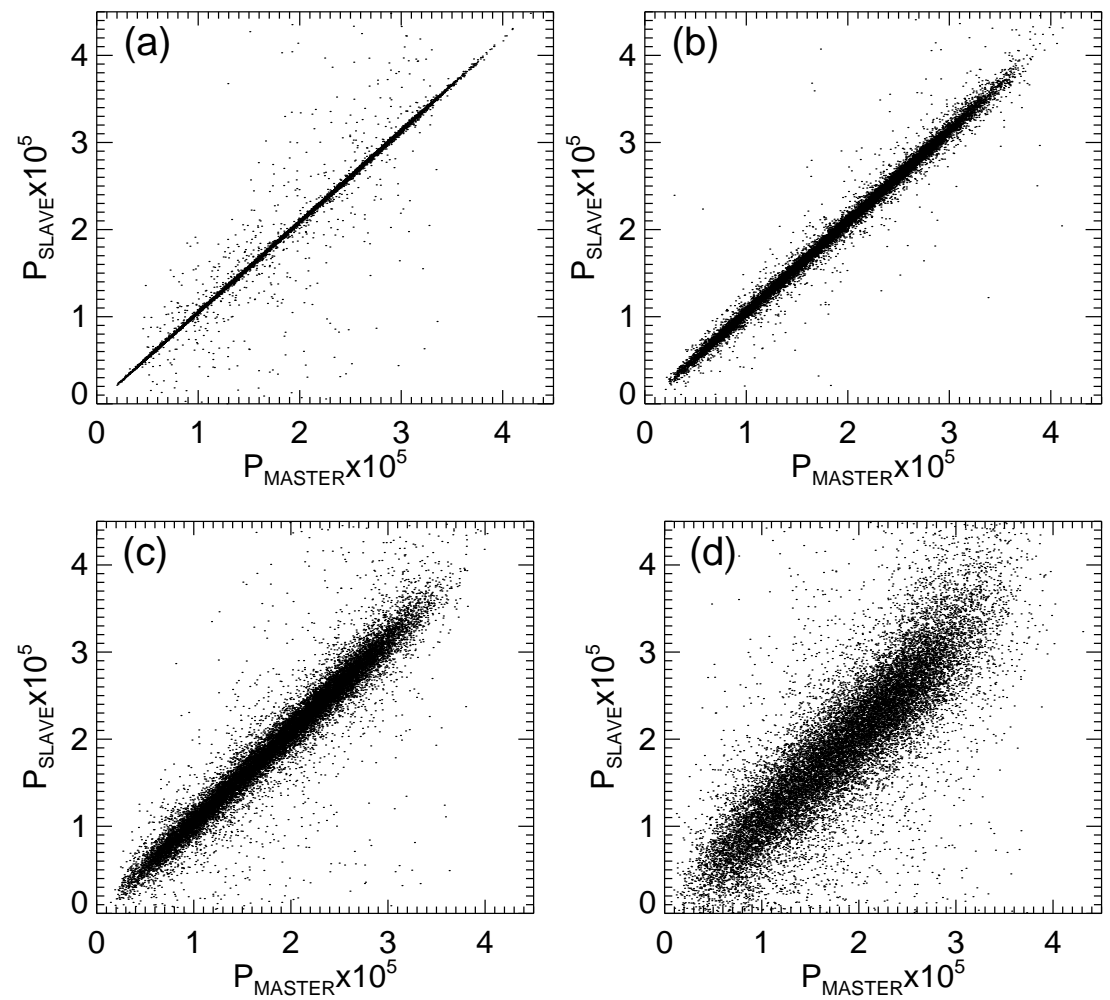

FIGURE 6. Synchronization diagram for different values of $\beta$, namely $\beta=0$ (a), $\beta=1 \times 10^{-6}$ $\mathrm{ns}^{-1}$ (b),$\beta=1 \times 10^{-5} \mathrm{~ns}^{-1}$ (c), and $\beta=1 \times 10^{-4} \mathrm{~ns}^{-1}$ (d). $\kappa_{r}=50 \mathrm{~ns}^{-1}, \Delta \omega=0, \tau=0.3 \mathrm{~ns}$ and the rest of parameters are those given in the previous.

in Ref. [15] using an iterative model, valid for weak to strong optical feedback, in the case of a Vertical Cavity Surface Emitting Laser (VCSEL). In this reference, the authors showed that there exists a restricted set of detunings, which form a synchronization window, that allows for a good synchronization. This window is roughly some tens of $\mathrm{GHz}$ wide and is asymmetric due to the linewidth enhancement factor parameter $\alpha$. The same effect is observed in our case using the rate equation model. The synchronization window also ranges roughly for $40 \mathrm{GHz}$, for the parameter values we have chosen, and is asymmetric due to the $\alpha$ parameter in such a way that a mirror image of Fig. 7 is obtained when changing the sign of $\alpha$. However, it is important to point out that in our case the detuning can be continuously varied within the synchronization window and the synchronization is still maintained. This fact is in contrast to the results presented in Ref. [15] where only specific values for the detuning, and small island around these values, allow for synchronization, even within the synchronization window. The discrepancy is due to the fact that in Ref. [15] the feedback phase $\omega_{t, r} \tau$ was fixed for both ML and SL at a constant value for all the detunings, while in our case this phase changes 

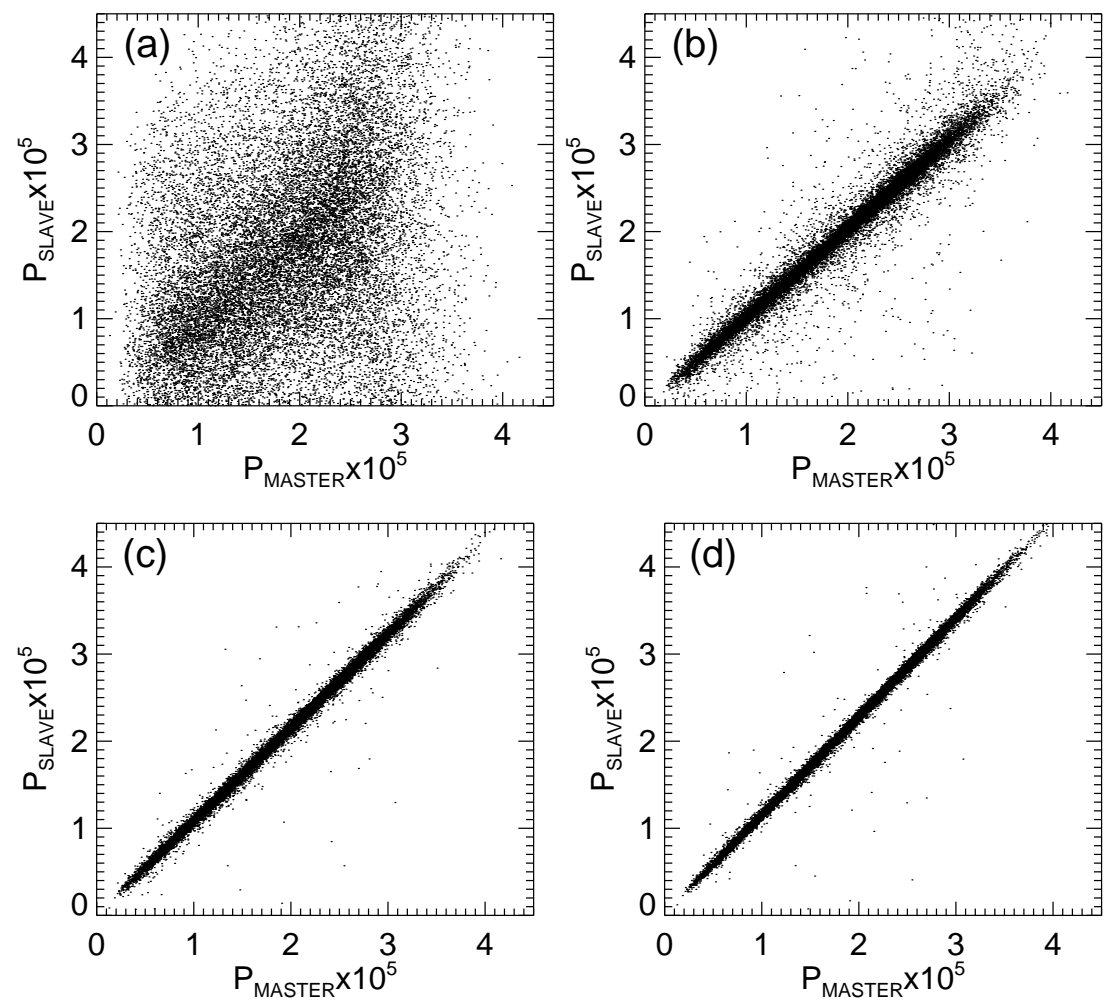

FIGURE 7. Synchronization diagram for different detunings $\Delta \nu=\Delta \omega /(2 \pi$, namely $\Delta \nu=-15$ $\mathrm{GHz}(\mathrm{a}), \Delta \nu=-5 \mathrm{GHz}$ (b), $\Delta \nu=5 \mathrm{GHz}$ (c), and $\Delta \nu=15 \mathrm{GHz}$ (d). $\kappa_{r}=50 \mathrm{~ns}^{-1}, \tau \omega=0.3 \mathrm{~ns}$ and the rest of parameters are those given in the previous section.

continuously with the detuning, accordingly to Eq. (1).

Finally, we analyze how the synchronization diagram changes with the feedback time $\tau$. From the mathematical point the phase space of a delayed system is infinite, and one should give an infinite number of initial conditions for the delayed feedback term when solving equation (1). This is why an attractor of a delay system can be of any, and even infinite, dimension. However, in practice this is not the case and it is well accepted that the number of degree of freedom associated with the ML and SL dynamics, i.e. the dimension of the chaotic attractors, increases with the feedback time $\tau$ and with the feedback coefficient $\gamma$. This point is important since one would expects that the higher the chaotic attractor dimension the better the confidentiality of the encrypted message. The feedback time $\tau$ can be easily varied by changing the position of the external mirrors. We have observed that the synchronization diagram degrades for increasing $\tau$, as shown in Fig. 8. To compensate this loss of synchronization $\kappa_{r}$ has to be increased. However, one would expect that the coupling between ML and SL has a maximum value in a real experiment. Once this limiting value is reached, the synchronization would be lost 

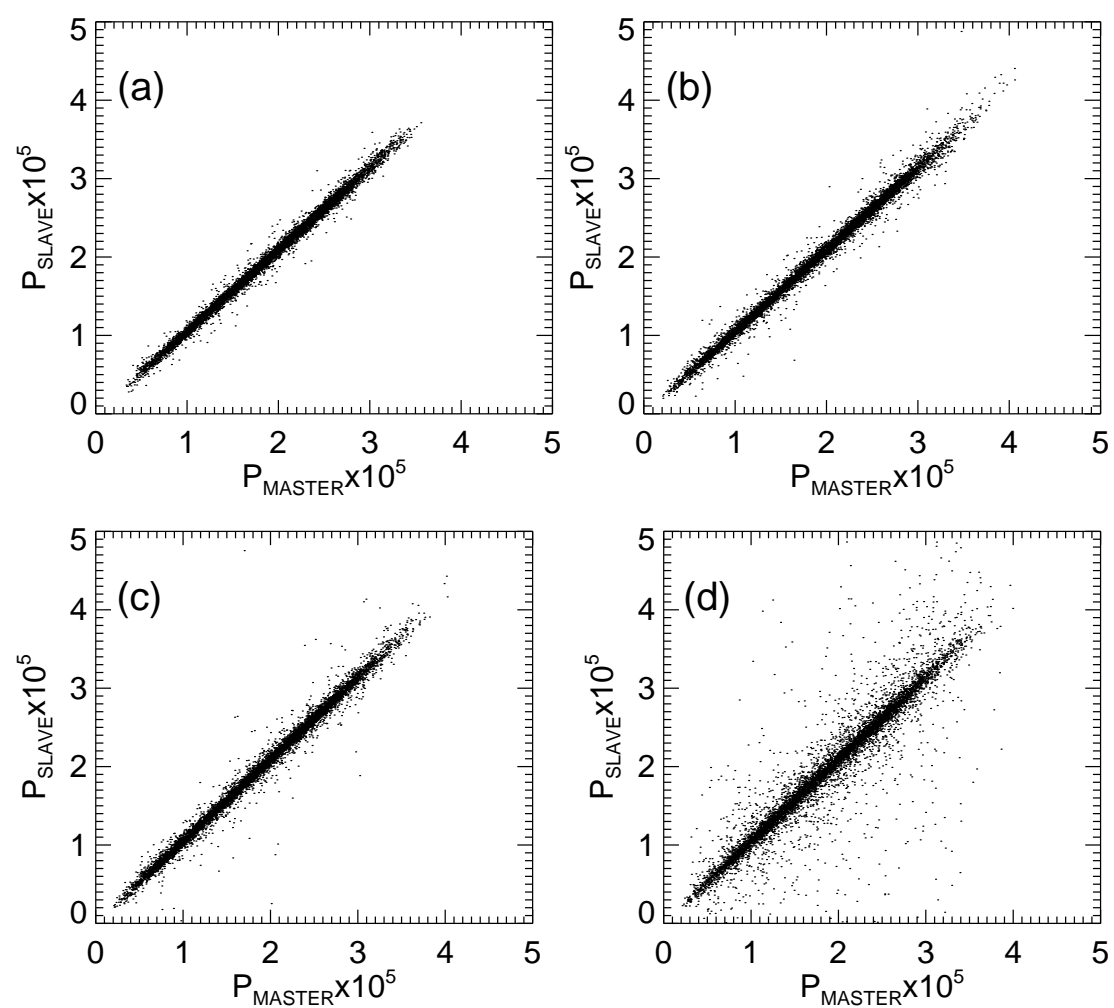

FIGURE 8. Synchronization diagram for $\tau=0.1 \mathrm{~ns}(\mathrm{a}), \tau=2 \mathrm{~ns}(\mathrm{~b}), \tau=10 \mathrm{~ns}$ )c), and $\tau=20 \mathrm{~ns}$ (d). $\kappa_{r}=50 \mathrm{~ns}^{-1}, \Delta \omega=0$ and the rest of parameters are those given in the previous section.

for larger values of $\tau$. A way to solve this problem is by using the set-up described in Refs. [11] and [13] where the SL operates without the external feedback. These two schemes offer the same filtering properties at the receiver and additionally they are more robust from the practical point of view.

\section{Message Encoding/Decoding}

In this part we show that, under good synchronization conditions, it is possible to encrypt and decrypt a message encoded within the chaotic carrier. For simplicity, we take identical parameters and operating conditions for both ML and SL, although slightly different lasers give rise to similar results.

As a first example we show in Fig. 9 the output of the ML and SL, and the encoded and decoded message, when a $1 \mathrm{GBit} / \mathrm{s}$ and $4 \mathrm{Gbit} / \mathrm{s}$ non-return to zero digital message is added at the output of the ML (chaos masking) following the technique used in Refs. $[5,6]$. When comparing the output of the ML with and without the message it is difficult to observe the fluctuations introduced by the en- 

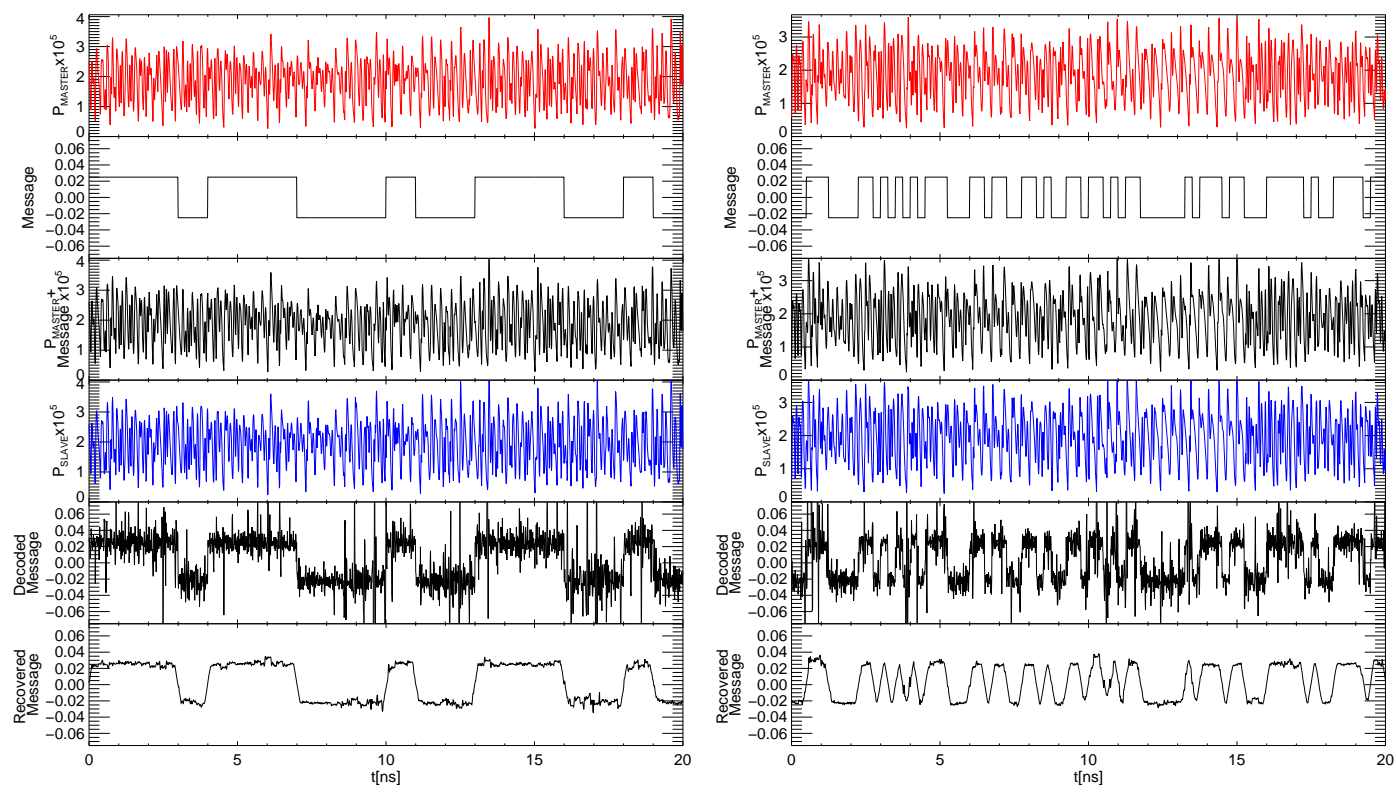

FIGURE 9. From top to bottom there is the output of the ML, the encoded message, the output of the ML with the message, the output of the SL, the decoded message, and the recovered message after filtering. $\tau=0.3 \mathrm{~ns}, \kappa_{r}=50 \mathrm{~ns}^{-1}, \Delta \omega=0$ and the rest of parameters are those given in the previous section. The message modulation rates are $1 \mathrm{Gbit} / \mathrm{s}$ (left panel) and 4 Gbit/s (right panel)

coded message. Also, optical spectra look very similar, as was shown in Ref. [6]. In both cases the recovery message exhibits fast oscillations that can be easily reduced by a simple filtering process. After this process, the recovered message compares quite well with the transmitted message. Also the synchronization diagram is good, as it is shown in Fig. 10 (left panel).

On the right panel of Fig. 10 the synchronization diagram can be seen when the message is included by modulating the injection current of the ML while keeping the other parameters fixed (chaos shift keying). Two parallel straight lines can be observed: one corresponds to the synchronization between ML and SL when a "1" bit is injected and the other when a " 0 " bit is injected. The separation between the two lines depends on the amplitude of the message and can be reduced by modulating the injection current with a smaller amplitude.

In Fig. 11 we show the ML and SL laser output together with the encoded and decoded message when a $1 \mathrm{Gbit} / \mathrm{s}$ and $4 \mathrm{Gbit} / \mathrm{s}$ non-return to zero digital message is encrypted. The quality of the recovered message after filtering is as good as the one obtained in the case of chaos masking (Figs. 9). However, this encoding technique is much simpler to implement in real systems. Moreover, it can be clearly seen that the message can be recovered very well with only one receiver which indicates that 

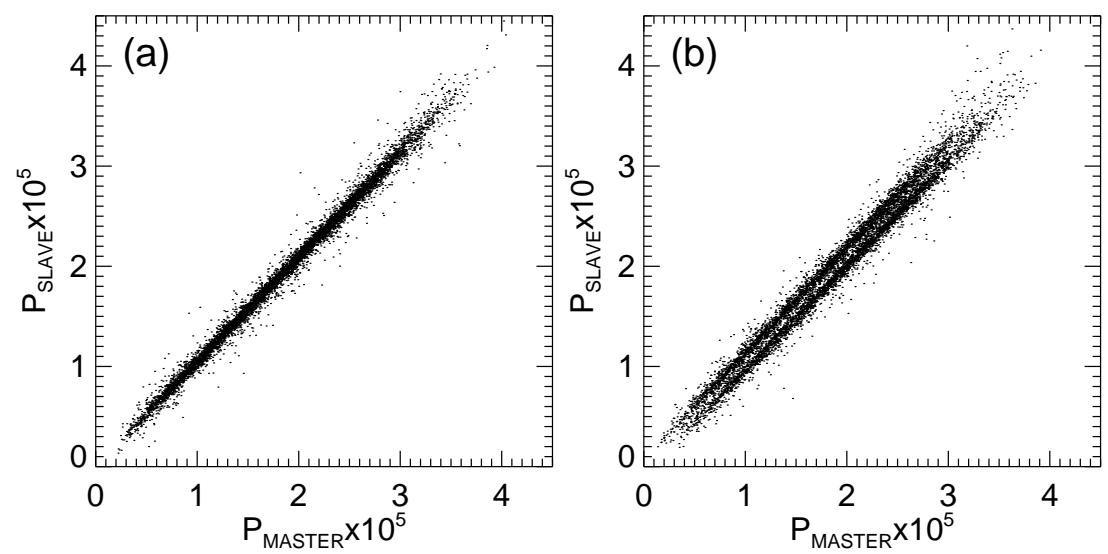

FIGURE 10. Synchronization diagram for the ML vs. SL when the message is added (chaos masking) at the output of the ML (a), and when the message is injected through the injection current (chaos shift keying) (b). $\tau=0.3 \mathrm{~ns}, \kappa_{r}=50 \mathrm{~ns}^{-1}, \Delta \omega=0$ and the rest of parameters are those given in the previous section.

two subsystems, one synchronizing to the " 1 " bit and the other one synchronizing to the "0" bit, suggested in Ref. [9] are not necessary.

\section{CONCLUSIONS}

In conclusion, we have numerically studied the synchronization of two chaotic semiconductor lasers with external optical feedback in a master-slave configuration. We have shown that a very good synchronization can be obtained when a minimum value for the coupling between the lasers is reached, even when the lasers are not identical. We have observed that the synchronization quality degrades with the noise, with the detuning between the ML and SL free running frequencies and with the dimension of the chaotic attractor (external-cavity round trip time and feedback coupling). It has been also shown that one the most critical parameter seems to be the detuning, although a good synchronization diagram can be continuously obtained for a detuning range, or detuning window, of tens of GHz. This detuning window is asymmetric due to the $\alpha$ factor.

We have also shown that a digital message modulated at frequencies up to 4 Gbit/s can be encoded in the chaotic signal of the master laser and recovered at the SL. The large and fast fluctuations that appear in the recovered message can be strongly reduced by a simple filtering process. We have shown that both chaos masking and chaos shift keying technique can be implemented and that both yield a similar quality of the recovery message, the chaos shift keying technique being the simplest one to implement in real systems. Moreover, we have also shown that, 

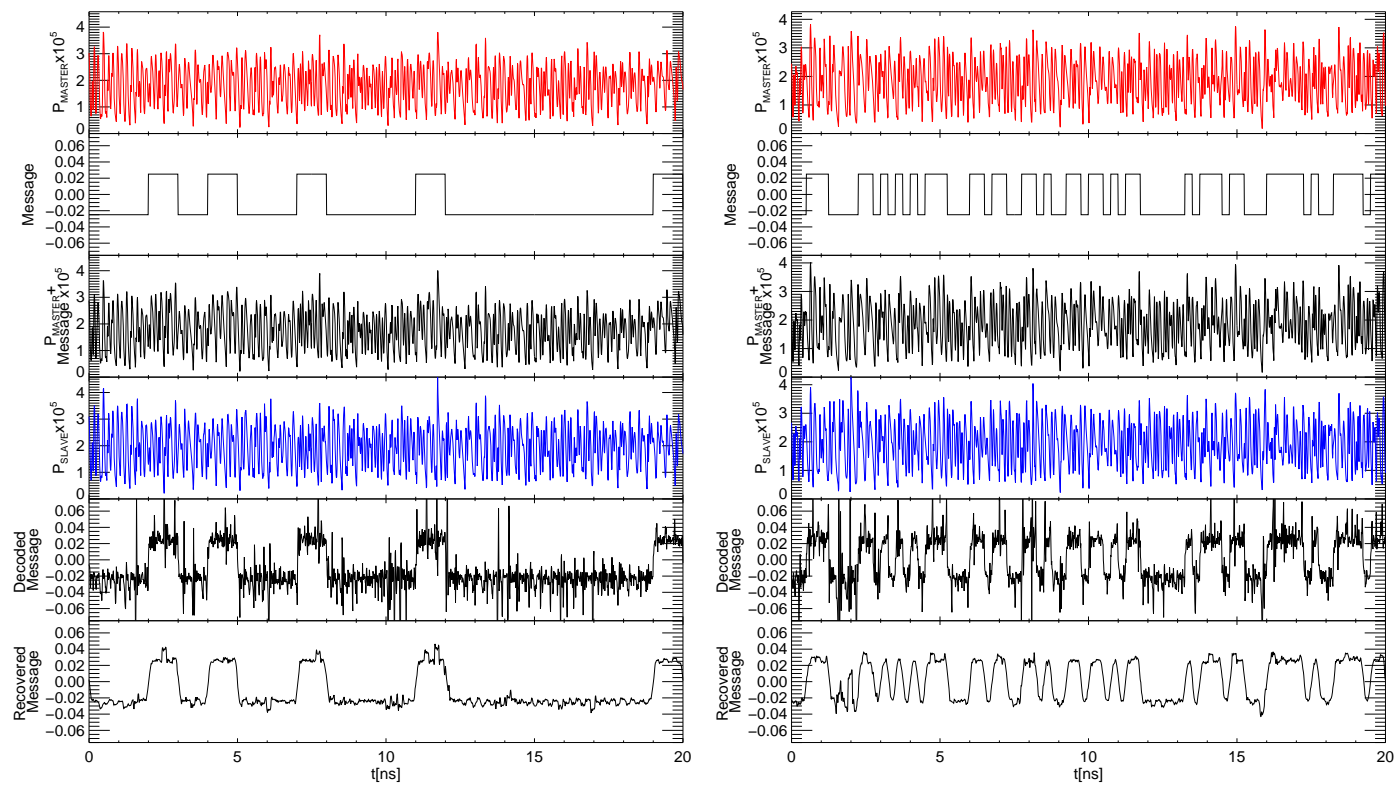

FIGURE 11. From top to bottom there is the output of the ML, the encoded message, the output of the ML with the message, the output of the SL, the decoded message, and the recovered message after filtering. $\tau=0.3 \mathrm{~ns}, \kappa_{r}=50 \mathrm{~ns}^{-1}, \Delta \omega=0$ and the rest of parameters are those given in the previous section. The message modulation rates are $1 \mathrm{Gbit} / \mathrm{s}$ (left panel) and 4 Gbit/s (right panel)

for the latter, only one receiver is necessary in contrast to previous studies.

To summarize, we have presented a simple but robust communication system based on chaotic synchronization that supports high modulation frequencies for encoded messages. This technique, understood as a codification at hardware level, could very well complement already existing encoding techniques, namely software codification.

\section{ACKNOWLEDGEMENTS}

I would like to specially thank Josep Mulet for helping with the simulations and figures, and to Pere Colet, Luis Pesquera and José Revuelta for fruitful discussions and valuable comments. This work has been supported by the CICYT, Spain, Project TIC99-0645-C05-02 and DGES, Spain, project PB97-0141-C02-01. 


\section{REFERENCES}

1. Pecora, L.M., and Carroll, T.L., "Synchronization in chaotic systems", Phys. Rev. Lett. 64, 821-823 (1990); "Driving systems with chaotic signals", Phys. Rev. A 44, 2374-2383 (1991).

2. Cuomo, K.M., and Oppenheim, A.V., "Circuit implementation of synchronized chaos with applications to communications", Phys. Rev. Lett. 71, 65-68 (1993); Cuomo, K.M., Oppenheim, A.V., and Strogatz, S.H., "Synchronization of Lorenz-based chaotic circuits with applications to communications", IEEE Trans. Circuits Sys. 40, 626-633 (1993).

3. Colet, P., and Roy, R., "Digital communications with synchronized chaotic lasers", Opt. Lett. 19, 2056-2058 (1994).

4. Van Wiggeren, G.D., and Roy, R., "Communication with chaotic lasers", Science 279, 1198-1200 (1998); "Chaotic communications using time delayed optical systems", Int. J. of Bif. and Chaos 9, 2129-2156 (1999).

5. Mirasso, C.R., Colet. P., and García-Fernández, P., "Synchronization of chaotic semiconductor lasers: application to encoded communications", Phot. Tech. Lett. 8, 299-301 (1996).

6. Sánchez-Díaz, A., Mirasso, C., Colet, P. and García-Fernández, P., "Encoded Gbit/s digital communications with synchronized chaotic semiconductor lasers", IEEE J. Quant. Electron. 35, 292-297 (1999).

7. Krauskopf, B., Wieczorek, S.M., and Lenstra, D., "Different types of chaos in optically injected semiconductor lasers", to appear in Appl. Phy. Lett.; (available via http://www.enm.bris.ac.uk/staff/berndk/publ.html)

8. Chen, H.F., and Liu, J.M., "Open loop chaotic synchronization of injection locked semiconductor lasers with Gigahertz range modulation", IEEE J. of Quant. Electron. 36, 27-34 (2000).

9. Annovazzi-Lodi, V., Donati, S., and Scire, A., "Synchronization of chaotic injectedlaser systems and its application to optical cryptography"", IEEE J. of Quant. Electron. 32, 953-959 (1996); "Synchronization of chaotic lasers by optical feedback for cryptographic applications", IEEE J. of Quant. Electron. 33, 1449-1454 (1997).

10. Rahman, L., Li, G., and Tian, F., "Remote synchronization of high frequency chaotic signals in semiconductor lasers for secure communications", Opt. Comm. 138, 91-94 (1997).

11. Goedgebuer, J.P., Larger, L., and Porte, H., "Optical cryptosystem based on synchronization of hyperchaos generated by a delayed feedback tunable laser diode", Phys. Rev. Lett. 80, 2249-2252 (1998); Larger, L., Goedgebuer, J.P., and Delorme, F., "Optical encryption system using hyperchaos generated by an optoelectronic wavelength oscillator", Phys. Rev. E 57, 6618-6624 (1998).

12. Sivaprakasam, S., and Shore, K.A., "Demonstration of optical synchronization of chaotic external-cavity laser diodes", Opt. Lett. 24, 466-468 (1999); "Message encoding and decoding using chaotic external-cavity diode lasers", IEEE J. Quant. Electron. 36, 35-39 (2000).

13. Fischer, I., Liu, Y., and Davis, P., "Synchronization of chaotic semiconductor laser dynamics on sub-ns timescales and its potential for chaos communication", to be 
published.

14. Spencer, P., Mirasso, C.R., Colet, P., and Shore, A., "Synchronization of chaotic VCSELs", IEEE J. Quant. Electron. 34, 1673-1679 (1998).

15. Spencer, P., and Mirasso, C.R., "Synchronization of chaotic VCSELs", IEEE J. Quant. Electron. 35, 803-809 (1999).

16. Uchida, A., Shinozuka, M., Ogawa, T., and Kannari, F., "Experiments on chaos synchronization in two separate microchip lasers", Opt. Lett. 24, 890-892 (1999). 